\title{
Three-Dimensional Inversion of Magnetotelluric Data for the Sediment-Basement Interface
}

\author{
Hongzhu Cai and Michael Zhdanov
}

\begin{abstract}
Determining the sediment-basement interface is the major step in evaluating the mineral resource potential of a region. The magnetotelluric (MT) method can be effectively used for solving this problem because there exists a strong contrast in resistivity between a conductive sedimentary basin and a resistive basement. Conventional inversions of MT data are aimed at determining the volumetric distribution of the conductivity within the inversion domain. The recovered distribution of the subsurface conductivity is typically diffusive, which makes it difficult to select the sediment-basement interface. This letter develops a novel approach to 3-D MT inversion for the depth-to-basement estimate. The key to this approach is selection of the model parameterization, with the depth to basement being the major unknown parameter. In order to estimate the depth to the basement, the inversion algorithm recovers both the thickness and the conductivities of the sedimentary basin. The forward modeling is based on the integral equation approach. The inverse problem is solved using a regularized conjugate gradient method. The Fréchet derivative matrix is calculated based on quasi-Born approximation. The developed method and the algorithm for MT inversion for the depth-to-basement estimate are illustrated on several realistic geoelectrical models.
\end{abstract}

Index Terms-Basement depth, integral equation (IE), inversion, magnetotelluric (MT).

\section{INTRODUCTION}

$\mathbf{T}$ HERE is a strong interest in developing effective geophysical methods for depth-to-basement estimation. Three main geophysical methods are used for this application-the seismic, potential field, and electromagnetic (EM) methods [1]-[4]. It is well known that seismic imaging is characterized by the highest resolution of the subsurface structures. However, in the case of complex near-surface heterogeneity (e.g., shallow, high-velocity, and highly heterogeneous basalt sills), typical for many frontier exploration regions, interpretation of seismic data represents a significant challenge, while using 3-D seismic surveys is very expensive [5]. These circumstances stimulated

Manuscript received June 21, 2015; revised November 25, 2015; accepted December 19, 2015. Date of publication January 13, 2016; date of current version February 24, 2016. This work was supported in part by The University of Utah Consortium for Electromagnetic Modeling and Inversion, TechnoImaging, and MFTI.

H. Cai is with the Consortium for Electromagnetic Modeling and Inversion, The University of Utah, Salt Lake City, UT 84112 USA (e-mail: caihongzhu@ hotmail.com)

M. S. Zhdanov is with Consortium for Electromagnetic Modeling and Inversion, The University of Utah, Salt Lake City, UT 84112 USA, TechnoImaging, Salt Lake City, UT 84107 USA, and also with the Moscow Institute of Physics and Technology (MIPT), 141700 Moscow, Russia (e-mail: michael. s.zhdanov@gmail.com).

Color versions of one or more of the figures in this paper are available online at http://ieeexplore.ieee.org.

Digital Object Identifier 10.1109/LGRS.2015.2512913 growing interest in using nonseismic geophysical methods, which could provide reasonable resolution but with lower cost [5].

Among the passive-source geophysical methods, potential field surveys have been widely used to estimate the depth to basement for decades (e.g., [3], [4], [6], and [7]). It is well known, however, that potential field data have relatively low resolution to the deep target. The EM data can provide higher resolution for subsurface formation than the gravity and magnetic data due to the frequency dependence of the EM field and the depth of investigation [8].

The magnetotelluric (MT) method is a passive-source EM method based on the analysis of the impedance tensor defined by the ratio of electric and magnetic fields [9], [10]. It has been demonstrated that this method can be used in exploration on local and regional scales [9]. The MT method also provides an effective approach for sedimentary basin analysis such as depth-to-basement estimation based on the conductivity contrast between the sediments and bedrocks [2], [5]. Conventional inversions of the MT data are usually aimed at determining the volumetric distribution of the conductivity within the inversion domain [8], [10], [11]. By the nature of the MT method, the recovered distribution of the subsurface conductivity is typically diffusive, although it can be focused by adopting more advanced regularization schemes such as focusing stabilizers [11].

In the problem of depth-to-basement estimation using geophysical data, the goal is to recover a sharp boundary between a sedimentary basin and a crystalline basement. Therefore, we need to adopt a sharp boundary parameterization of the subsurface for the inversion. This type of parameterization has been widely used for potential field inversion to recover the depth to basement (e.g. [3], [4], and [7]). These parameterization approaches, however, have not been widely used for MT inversion problems, especially in the 3-D case. The main reason is that it is difficult to find a straightforward relationship between the EM data and the depth to the basement in a sharp boundary parameterization. Chen et al. [12] introduced a stochastic inversion algorithm for a 2-D MT inverse problem to recover the location of the sediment-basement interface and also the conductivities within each layer. However, this method cannot be easily extended to a 3-D case because of the high computational cost of stochastic inversion.

In this letter, we introduce a parameterization for the MT inversion, similar to the discretization used in GallardoDelgado et al. [7] for potential field inversion. For simplicity, it is assumed that the subsurface comprises a conductive layer of sediments and a resistive bedrock foundation. The interface between the sediments and the bedrock has an arbitrary shape. 


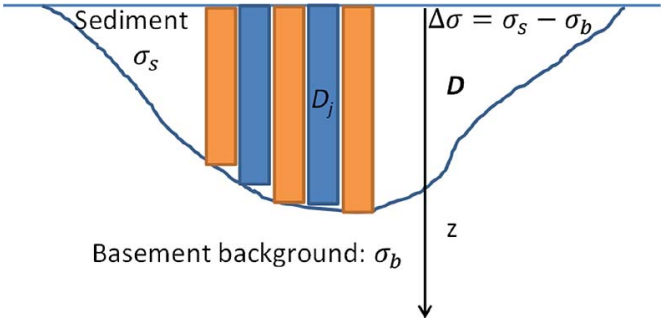

Fig. 1. Sketch of a conductivity model of the sedimentary basin. Domain $D$ represents the conductive sediments with conductivity $\sigma_{s}$, which is discretized into a grid of vertical columns.

The sediment packs are discretized into a grid of columns with known horizontal dimensions. The MT response of the geoelectrical model is computed using the integral equation (IE) method. The advantage of the IE approach is that, in this case, the Fréchet derivatives of the data with respect to the columns' thickness and the sediment's conductivity could be calculated in closed form using the IE representation based on the quasi-Born approximation. A realistic model study shows that the developed method can be used for fast and accurate estimation of the depth to basement using MT data.

\section{REgularized InVERsion BASED On the IE Method}

The methodology of a 3-D contraction IE method for geophysical EM modeling was discussed in detail by Zhdanov [8], [11]. We present here the basic equations of the IE method for completeness. In the framework of the IE formulation, the total electric and magnetic fields are decomposed into their background $\left(\mathbf{E}^{b}, \mathbf{H}^{b}\right)$ and anomalous $\left(\mathbf{E}^{a}, \mathbf{H}^{a}\right)$ parts [8], [11], with the anomalous fields expressed as an integral of the excess currents within domain $D$ with anomalous conductivity, $\Delta \sigma$, as follows:

$$
\begin{aligned}
\mathbf{E}^{a}\left(\mathbf{r}_{j}\right) & =\iiint_{D} \hat{\mathbf{G}}_{E}\left(\mathbf{r}_{j} \mid \mathbf{r}\right) \Delta \sigma(\mathbf{r}) \cdot\left[\mathbf{E}^{b}(\mathbf{r})+\mathbf{E}^{a}(\mathbf{r})\right] d v \\
\mathbf{H}^{a}\left(\mathbf{r}_{j}\right) & =\iiint_{D} \hat{\mathbf{G}}_{H}\left(\mathbf{r}_{j} \mid \mathbf{r}\right) \Delta \sigma(\mathbf{r}) \cdot\left[\mathbf{E}^{b}(\mathbf{r})+\mathbf{E}^{a}(\mathbf{r})\right] d v
\end{aligned}
$$

where $\mathbf{G}_{E}\left(\mathbf{r}_{j} \mid \mathbf{r}\right)$ and $\mathbf{G}_{H}\left(\mathbf{r}_{j} \mid \mathbf{r}\right)$ are the electric and magnetic Green's tensors defined for a medium with the background conductivity $\sigma_{b}$.

Formula (1) becomes an IE if $\mathbf{r}_{j} \in \mathbf{r}$. The anomalous electric field inside domain $D$ can be obtained by solving the IE. Once the electric field in domain $D$ is found, the anomalous field at the receivers outside domain $D$ can be calculated using the aforementioned two formulas. The components of the MT impedance tensor are then computed using the known linear relationships between the horizontal components of the electric and magnetic fields [9], [10].

In order to formulate a corresponding inverse MT problem, we consider a model of the sedimentary basin shown in Fig. 1. The basement has the background conductivity $\sigma_{b}$, and domain $D$ represents the conductive sediments. We assume for simplicity that the sediments have a uniform conductivity of $\sigma_{s}$; however, in a general case, the method can be extended to the case of an arbitrary distribution of the conductivity

$$
\sigma_{s}(\mathbf{r})=\sigma_{b}(\mathbf{r})+\Delta \sigma(\mathbf{r}) .
$$

In the inversion, domain $D$ is discretized into $N$ columns, denoted as subdomains $D_{j}$, with conductivity $\sigma_{s}$. The horizontal dimension of each subdomain is known and fixed. Contrary to the conventional MT inversion, which recovers a volumetric distribution of the subsurface conductivities, the goal is to find the depth of each column. If the conductivity of the sediments is unknown, the inversion can also recover $\sigma_{s}(\mathbf{r})$ jointly with the depth-to-basement estimate. We should note that, for IE forward modeling, the columns should be further discretized in the vertical direction.

The inversion of MT data is an ill-posed problem. In order to obtain a stable and geologically reasonable result, one has to apply regularization to impose some restrictions on the solution. The regularized inversion is based on the minimization of the Tikhonov parametric functional [14]

$$
\begin{array}{r}
P(\mathbf{m}, \mathbf{d})=\left(\mathbf{W}_{d} \mathbf{A}(\mathbf{m})-\mathbf{W}_{d} \mathbf{d}\right)^{*}\left(\mathbf{W}_{d} \mathbf{A}(\mathbf{m})-\mathbf{W}_{d} \mathbf{d}\right) \\
+\left(\mathbf{W}_{m} \mathbf{m}-\mathbf{W}_{m} \mathbf{m}_{\mathrm{apr}}\right)^{T}\left(\mathbf{W}_{m} \mathbf{m}-\mathbf{W}_{m} \mathbf{m}_{\mathrm{apr}}\right)
\end{array}
$$

where $\mathbf{A}$ is the forward modeling operator, $\mathbf{d}$ is the vector of the observed data, $\mathbf{W}_{d}$ is a diagonal data weighting matrix, $\mathbf{m}$ is the vector of the model parameters, $\mathbf{m}_{\text {apr }}$ is the a priori model of the depth to the basement, and " $*$ " is the symbol of complex conjugate transposition.

A diagonal matrix of the model parameter weights $\mathbf{W}_{m}$ is calculated based on the integrated sensitivity as follows [8]:

$$
\mathbf{W}_{m}=\operatorname{diag}\left(\mathbf{F}^{T} \mathbf{F}\right)^{\frac{1}{2}}
$$

where $\mathbf{F}$ is the Fréchet derivative matrix. The minimization is based on the reweighted regularized conjugate gradient (RCG) method [11].

This algorithm involves the matrix multiplication only. Therefore, the computational complexity of the developed inversion method depends primarily on the complexity of the forward modeling operation. We use the fully parallelized EM forward modeling software PIE3D, developed by the Consortium for Electromagnetic Modeling and Inversion (CEMI), which is designed for distributed memory machines. The details of the numerical scheme implemented in PIE3D can be found in related publications [8], [11], [13].

\section{Frechet Derivative Calculations}

One key aspect of nonlinear inversion using the conjugate gradient method is the need for computing the Fréchet derivative of the observed data with respect to the thickness of the column and the sediment conductivity $\sigma_{s}$. The most effective way of solving this problem in the framework of the IE method is using the quasi-Born approximation [8], which provides a reasonable estimation of the Fréchet derivative for the depthto-basement inversion.

First, we will derive the expression of the Fréchet derivative with respect to the thickness of the column with a given sediment conductivity $\sigma_{s}$. Consider the sediment-basement interface model shown in Fig. 1 with a column discretization. The anomalous field in the receiver's positions $\mathbf{r}_{i}$ can be calculated according to formula (1) as follows:

$$
\mathbf{E}^{a}\left(\mathbf{r}_{i}\right)=\iiint_{D} \hat{\mathbf{G}}_{E}\left(\mathbf{r}_{i} \mid \mathbf{r}\right) \cdot[\Delta \sigma(\mathbf{r}) \mathbf{E}(\mathbf{r})] d v=\sum_{j=1}^{N} \mathbf{E}_{j}^{a}\left(\mathbf{r}_{i}\right)
$$


where $\mathbf{E}(\mathbf{r})$ is the total electric field within anomalous domain $D$ and $\mathbf{E}_{j}^{a}\left(\mathbf{r}_{i}\right)$ represents the anomalous field at receiver $\mathbf{r}_{i}$ contributed from the $j$ th column $D_{j}$ of the sediments' pack

$$
\mathbf{E}_{j}^{a}\left(\mathbf{r}_{i}\right)=\iiint_{D_{j}} \hat{\mathbf{G}}_{E}\left(\mathbf{r}_{i} \mid \mathbf{r}\right) \cdot[\Delta \sigma(\mathbf{r}) \mathbf{E}(\mathbf{r})] d v .
$$

The volume integral in the last formula can be written explicitly as a combination of the surface integral over the horizontal section of the $j$ th column $\left(S_{j}\right)$ and a linear integral along the vertical coordinate from the surface $(z=0)$ down to the bottom of the $j$ th column $\left(z=z_{j}\right)$

$\mathbf{E}_{j}^{a}\left(\mathbf{r}_{i}\right)=\int_{0}^{z_{j}}\left\{\iint_{S_{j}} \widehat{\mathbf{G}}_{E}\left(\mathbf{r}_{i} \mid(x, y, z)\right) \cdot[\Delta \sigma(\mathbf{r}) \mathbf{E}(x, y, z)] d x d y\right\} d z$.

The Fréchet derivative of the anomalous field to the thickness $z_{j}$ of the $j$ th column can be calculated by considering the variation $\delta \mathbf{E}^{a}\left(\mathbf{r}_{i}\right)$ of the anomalous electric field with respect to variations $\delta z_{j}$ of the depth of the $j$ th column as follows:

$$
F_{i j}=\frac{\delta \mathbf{E}^{a}\left(\mathbf{r}_{i}\right)}{\delta_{z_{j}}}=\frac{\delta \sum_{k=1}^{N} \mathbf{E}_{k}^{a}\left(\mathbf{r}_{i}\right)}{\delta_{z_{j}}}=\frac{\delta \mathbf{E}_{j}^{a}\left(\mathbf{r}_{i}\right)}{\delta_{z_{j}}} .
$$

Taking into account (8) and using the concept of quasi-Born approximation [8], the Fréchet derivative in (9) can be reduced to the following expression:

$$
F_{i j}=\iint_{S_{j}} \widehat{\mathbf{G}}_{E}\left(\mathbf{r}_{i} \mid\left(x, y, z_{j}\right)\right) \cdot[\Delta \sigma \mathbf{E}(x, y, z)] d x d y .
$$

Expression (10) requires knowledge of the total electric field $\mathbf{E}(x, y, z)$ within the anomalous domain. On the first iteration of the inversion, we may substitute the background electric field $\mathbf{E}^{b}\left(x, y, z_{j}\right)$ for the total electric field, just arriving at the conventional Born approximation for the Fréchet derivative calculation. However, on iteration number $n$, following Zhdanov [8], one can use a quasi-Born approximation, which is based on substituting the total electric field $\mathbf{E}^{(n)}$ computed for the current iteration, for the unknown total electric field $\mathbf{E}$ in formula (10), as follows:

$$
F_{i j}^{(n)}=\iint_{S_{j}} \widehat{\mathbf{G}}_{E}\left(\mathbf{r}_{i} \mid\left(x, y, z_{j}\right)\right) \cdot\left[\Delta \sigma \mathbf{E}^{(n)}\left(x, y, z_{j}\right)\right] d x d y .
$$

The advantage of using the quasi-Born approximation is that it provides an explicit expression for the Fréchet derivative, which is at the same time very accurate due to the presence of the total electric field $\mathbf{E}^{(n)}$, estimated at the current iteration, in formula (11). Note also that the accuracy of the quasi-Born approximation increases with the iteration number because we obtain a more accurate approximation $\mathbf{E}^{(n)}$ of the total field inside the anomalous domain. The surface integrals in formula (11) are computed numerically with high accuracy using a fine discretization of the column in the $x$ and $y$ directions.

In a case of inversion for both the column's thickness and the sediment's conductivities, we need to determine the Fréchet derivative with respect to conductivity. This expression can also (a)

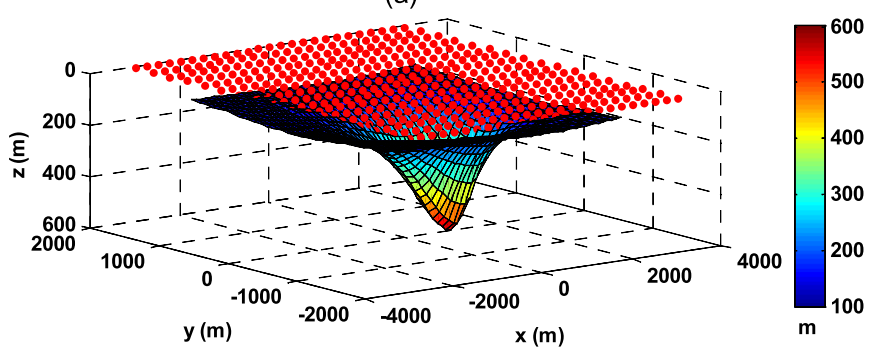

(b)

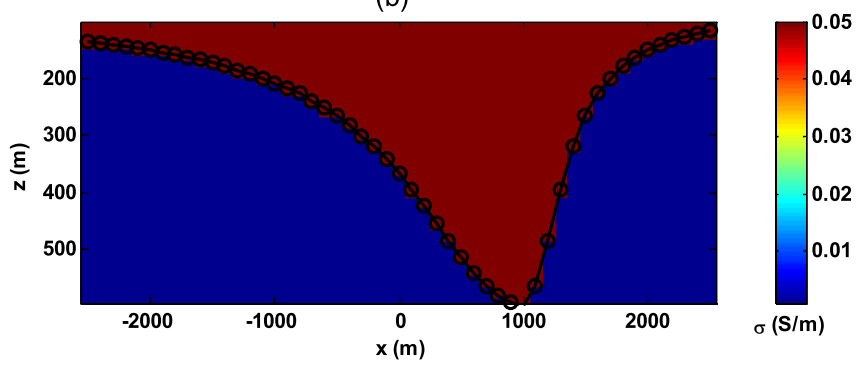

Fig. 2. Synthetic sediment-basement interface model with asymmetric shape Panel (a) shows a 3-D view of the sediment-basement interface model, with red dots indicating the MT receivers on the Earth's surface. The color scale represents the depth of the sediment-basement interface. Panel (b) shows a vertical cross section at $y=0$ of model 1 . The black line with circles indicates the actual sediment-basement interface, while the prismatic approximation of the interface is shown by the two different colors, reflecting the conductivity of the sediments of $0.05 \mathrm{~S} / \mathrm{m}$ on the corresponding color scale.

be found in a similar way using the quasi-Born approximation [8]. The Fréchet derivatives for the magnetic fields can also be derived in a similar way.

For MT inversion, the input data are the components of the impedance tensor, which involve the combinations of electric and magnetic fields. The Fréchet derivatives for the impedances can be obtained by using the relationships between the impedances and the EM fields with two different polarizations. The analytical expressions of the Fréchet derivatives of the impedance tensor can be found in Zhdanov [8].

\section{Model Studies}

We will illustrate our inversion algorithm using several realistic synthetic models of the sediment-basement interface. In the model study, we consider the inversion for both the depth to the basement and the conductivity of the sediments. We should note, however, that in practical applications, one should apply a conventional 3-D inversion of the MT data first in order to determine the volumetric distribution of the conductivity in the subsurface. The model produced by the conventional MT inversion can be used to create the initial model for the depthto-basement estimate using the developed novel algorithm.

Model 1 represents a sediment-basement interface with a maximum depth of $600 \mathrm{~m}$. The conductivity of the basement is $0.001 \mathrm{~S} / \mathrm{m}$, while the conductivity of the sediments is $0.05 \mathrm{~S} / \mathrm{m}$. Fig. 2(a) shows a 3-D model view of the sediment-basement interface model with MT receivers located on the Earth's surface. The survey covers the area from -3000 to $3000 \mathrm{~m}$ in the $x$ direction and from -2000 to $2000 \mathrm{~m}$ in the $y$ direction with $250-\mathrm{m}$ receiver and line spacing. The size of the columns used to discretize the inversion domain is $125 \mathrm{~m}$ in both the 

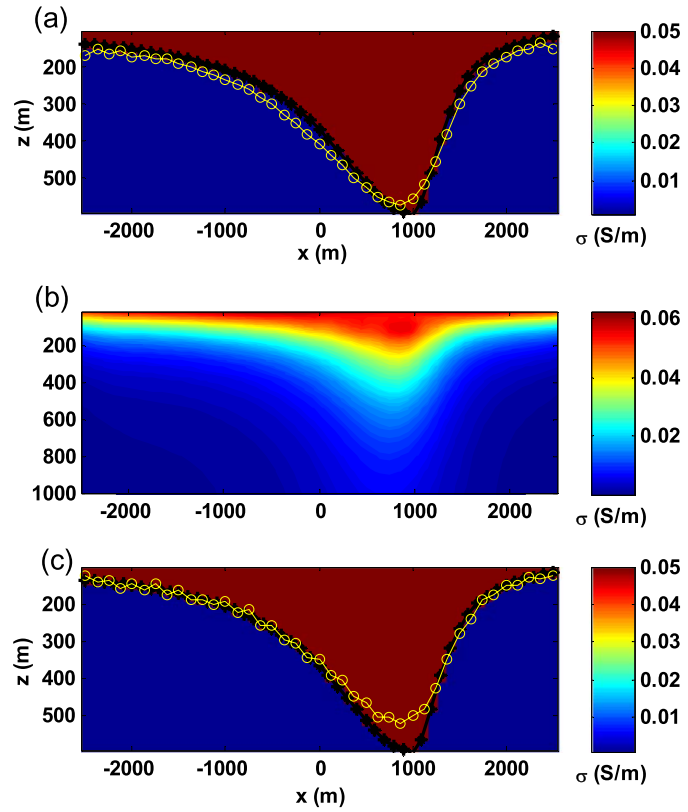

Fig. 3. Inversion result for model 1 at $y=0$. Panel (a) shows the inversion result of the sediment-basement interface with a flat surface as initial model. Panel (b) shows a vertical section of the subsurface conductivity distribution recovered from the conventional MT inversion method. Panel (c) shows the inversion result for the sediment-basement interface, with the starting model created from the conventional MT inversion in panel (b). The circles in panels (a) and (c) represent the recovered model, and the stars show the true sediment-basement interface.

$x$ and $y$ directions. Fig. 2(b) shows a vertical cross section of the conductivity distribution for this model.

We used nine frequencies uniformly distributed from 0.01 to $100 \mathrm{~Hz}$ in logarithmic space, and the data were contaminated by $5 \%$ random noise. Based on the experience, at least two frequencies per decade is required to recover both the depth to the basement and the sediment's conductivity in the model.

In the first numerical experiment, we assume that there is no a priori information for the sediment-basement interface and the sediment conductivity. We have used a flat surface at $300 \mathrm{~m}$ depth and sediment conductivity of $0.1 \mathrm{~S} / \mathrm{m}$ as the initial model. We run 80 iterations of the RCG method until the misfit reaches $5 \%$. We used adaptive regularization, with the regularization parameter updated in every iteration [11]. The selected regularization parameter should be able to balance the misfit and stabilizer functionals. The too large regularization parameter will slow the convergence, while the too small parameter can produce a result, which is not geologically meaningful. Adaptive regularization automatically selects an optimal value of the regularization parameter [11], [13]. Fig. 3(a) shows a vertical section of the inversion result, with the yellow circles representing the recovered model and the black stars indicating the true sediment-basement interface. The maximum depth recovered from the inversion was $573 \mathrm{~m}$, while the actual maximum depth of the sedimentary basin was $600 \mathrm{~m}$. The inverted sediment conductivity converged to the value of $0.0437 \mathrm{~S} / \mathrm{m}$, which was very close to the true value of $0.05 \mathrm{~S} / \mathrm{m}$.

We have also applied the conventional 3-D MT inversion for this model to get a volumetric distribution of the subsurface conductivity distribution. Fig. 3(b) shows a vertical section of the recovered conductivity distribution at $y=0$.
One can see that the shape of the sedimentary basin and the sediment conductivity is roughly recovered. However, it is hard to determine the sediment-basement interface since the conductivity distribution recovered from the conventional MT inversion is very diffusive. We have approximately determined the sediment-basement interface from the conventional MT inversion. We use this interface and the estimated sediment conductivity $(0.03 \mathrm{~S} / \mathrm{m})$ from the conventional MT inversion as the initial model for the depth-to-basement inversion. Fig. 3(c) shows a vertical section of the inversion result, with the yellow circles representing the recovered model and the black stars indicating the true sediment-basement interface. One can see that the recovered sediment-basement interface is very close to the true model. The inverted sediment conductivity converged to the value of $0.0454 \mathrm{~S} / \mathrm{m}$, which was very close to the true value of $0.05 \mathrm{~S} / \mathrm{m}$.

By comparing Fig. 3(a) and (c), one can see that the location of the sediment-basement interface recovered from the developed method with the initial model created from the conventional MT inversion [Fig. 3(c)] is much closer to the true model. Moreover, the final recovered sediment conductivity in Fig. 3(c) is also much closer to its true value. In addition, we should note that the inversion result shown in Fig. 3(a) required 80 iterations, while the inverse model shown in Fig. 3(c) was obtained after 30 iterations only.

The conventional MT inversion result in Fig. 3(b) took $12 \mathrm{~h}$ on 12 core workstation, whereas our depth-to-basement inversion took $1 \mathrm{~h}$ only since $2-\mathrm{D}$ discretization of the sedimentbasement interface only is required in our method.

\section{INVERSION OF THE MT DATA FOR A USGS BASIN MODEL}

In this section, we consider the inversion of MT data computer simulated for the USGS basin model (Big Bear Lake basin). The Big Bear Lake area is located in the southeast part of California. It is characterized by deep sediment basins surrounded by uplifted bedrocks. The basin was well studied by using collected gravity anomaly data [15]. Cai and Zhdanov [4] also inverted the depth to basement in this area using the released gravity data. However, the MT data were not available in this area.

We computer simulated the synthetic MT data at 441 MT stations located on a rectangular grid at 9 frequencies ranging from 0.01 to $100 \mathrm{~Hz}$ using the basin model that was produced by the gravity inversion [4]. A 5\% random noise was added to the synthetic data as well. In the USGS basin model, the conductivities of the basement and sediments were selected as 0.001 and $0.05 \mathrm{~S} / \mathrm{m}$, respectively. The inversion was done for the depth-to-basement estimate and the sediment conductivity. The inversion process was terminated after 30 iterations, when the misfit between the observed and predicted data reached the noise level.

Fig. 4 shows a comparison of the maps of the true model and the inversion result. One can see that the geometry of the USGS basin model was reconstructed very well. The recovered maximum depth of the basin was $830 \mathrm{~m}$, which was very close to the actual maximum depth of $850 \mathrm{~m}$. Fig. 5 presents the vertical sections of the inversion results along two profiles ( $y=-1300 \mathrm{~m}$ and $y=200 \mathrm{~m}$ ), shown by the dashed white 

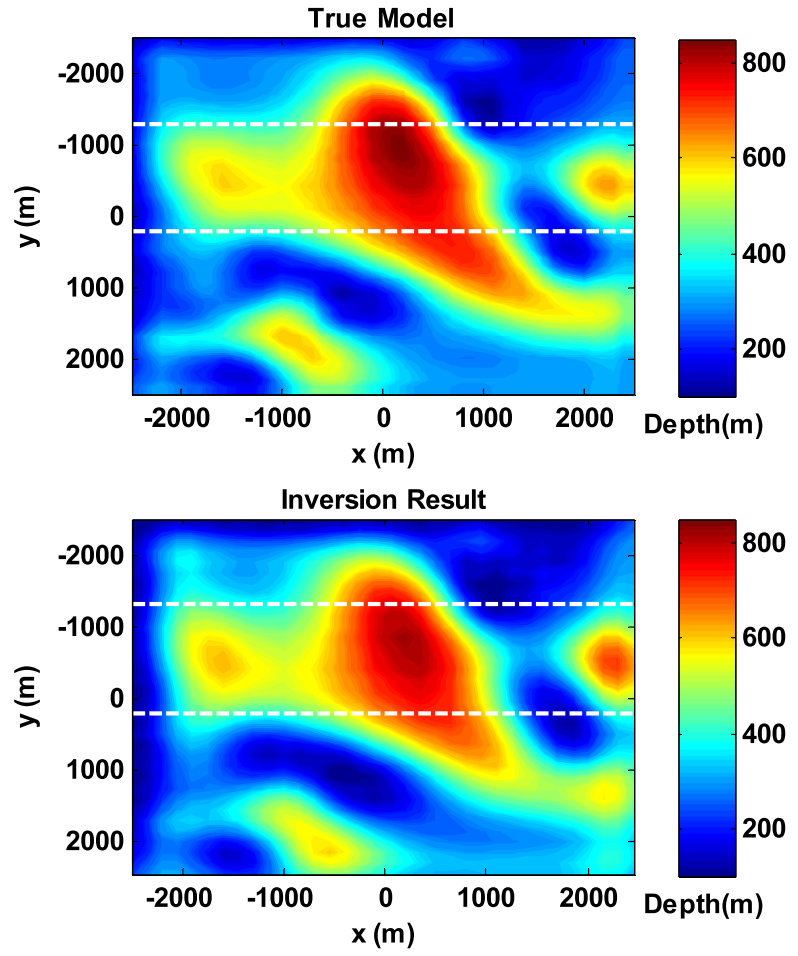

Fig. 4. Maps of the true model of the sediment-basement interface (top panel) and of the inversion result (bottom panel) for the USGS model. The dashed white lines show two selected profiles at $y=-1300 \mathrm{~m}$ and $y=200 \mathrm{~m}$, respectively.
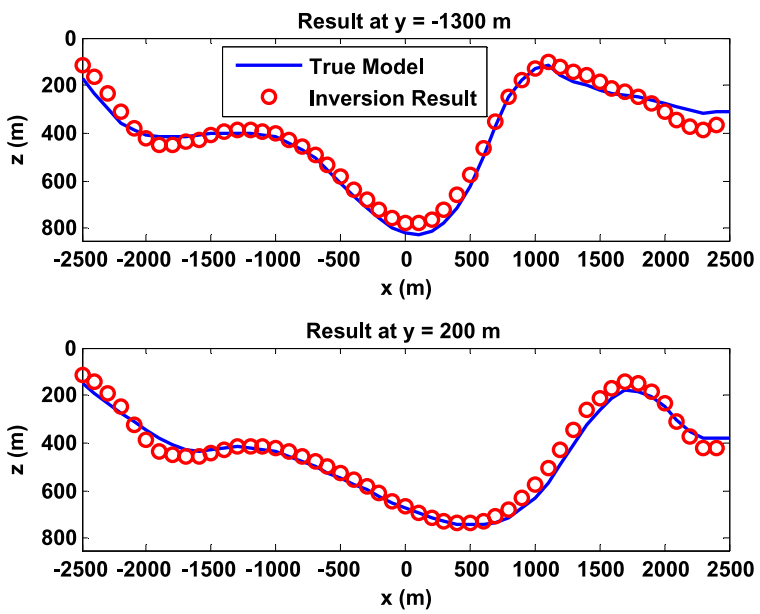

Fig. 5. Comparison of the true interface and inversion result for the USGS model at the profiles $y=-1300 \mathrm{~m}$ and $y=200 \mathrm{~m}$. The solid curve shows the true model, while the circles represent the inversion result.

lines in Fig. 4. One can see that the inversion did a good job in determining the correct interface between the sedimentary basin and the basement. The final recovered sediment conductivity is $0.059 \mathrm{~S} / \mathrm{m}$, which is also very close to its true value.

\section{CONCLUSION}

We have developed a novel approach to the inversion of the MT data for the depth to the basement. The key component of this approach is selection of the model parameterization, with the depth to the basement being the major unknown parameter. An effective and accurate method of computing the Fréchet derivatives with respect to the depth to the basement has been introduced based on the quasi-Born approximation of the anomalous EM fields.

The proposed method provides a fast imaging technique for recovering a surface with the sharp conductivity contrast using the MT data. Comparing to the conventional methods, the developed method produces a clearer image of the conductivity contrast surface. The method works well for the sedimentary basin environment without a significant localized anomaly.

The developed method and computer code were tested using several typical sedimentary basin models. The numerical studies have also demonstrated that the MT inversion can simultaneously recover both the thickness of the sedimentary basin and its conductivity.

\section{ACKNOWLEDGMENT}

The authors would like to thank Consortium for Electromagnetic Modeling and Inversion (CEMI) and the Center for High Performance Computation at The University of Utah for the cluster allocation.

\section{REFERENCES}

[1] Afnimar, K. Koketsu, and K. Nakagawa, "Joint inversion of refraction and gravity data for the three-dimensional topography of a sedimentbasement interface," Geophys. J. Int., vol. 151, no. 1, pp. 243-254, Oct. 2002.

[2] I. Zevallos, M. Assumpção, and A. L. Padilha, "Basement depth in the Paraná basin from joint inversion of teleseismic receiver functions and magnetotelluric sounding," in Proc. I Simpósio Brasileiro Geofísica, 2004, pp. 26-28.

[3] H. Cai and M. S. Zhdanov, "Modeling and inversion of magnetic anomalies caused by sediment-basement interface using three-dimensional Cauchy-type integrals," IEEE Geosci. Remote Sens. Lett., vol. 12, no. 3, pp. 477-481, Mar. 2015.

[4] H. Cai and M. S. Zhdanov, "Application of Cauchy-type integrals in developing effective methods for depth-to-basement inversion of gravity and gravity gradiometry data," Geophysics, vol. 80, no. 2, pp. G81-G94, Mar. 2015

[5] B. Tournerie and M. Chouteau, "Three-dimensional magnetotelluric survey to image structure and stratigraphy of a sedimentary basin in Hungary," Phys. Earth Planet. Interiors, vol. 150, no. 1-3, pp. 197-212, May 2005.

[6] V. Barbosa, V. J. Silva, and W. Medeiros, "Gravity inversion of basement relief using approximate equality constraints on depths," Geophysics, vol. 62, no. 6, pp. 1745-1757, Nov./Dec. 1997.

[7] L. A. Gallardo, M. A. Perez, and E. G. Treviño, "A versatile algorithm for joint 3D inversion of gravity and magnetic data," Geophysics, vol. 68, no. 3, pp. 949-959, May 2003.

[8] M. S. Zhdanov, Geophysical Electromagnetic Theory and Methods. Amsterdam, The Netherlands: Elsevier, 2009.

[9] M. S. Zhdanov and G. Keller, The Geoelectrical Methods in Geophysical Exploration. Amsterdam, The Netherlands: Elsevier, 1994

[10] M. N. Berdichevsky and V. L. Dmitriev, Models and Methods of Magnetotellurics. Berlin, German: Springer-Verlag, 2008.

[11] M. S. Zhdanov, Geophysical Inverse Theory and Regularization Problems. Amsterdam, The Netherlands: Elsevier, 2002.

[12] J. Chen, G. Hoversten, K. Key, G. Nordquist, and W. Cumming, "Stochastic inversion of magnetotelluric data using a sharp boundary parameterization and application to a geothermal site," Geophysics, vol. 77, no. 4, pp. E265-E279, Jul. 2012.

[13] M. S. Zhdanov, Inverse Theory and Applications in Geophysics. Amsterdam, The Netherlands: Elsevier, 2015.

[14] A. N. Tikhonov and V. Y. Arsenin, Solutions of Ill-Posed Problems. Washington, DC, USA: V. H. Winston and Sons, 1977.

[15] C. Roberts, R. Jachens, A. Katzenstein, G. Smith, and R. Johnson, "Gravity map and data of the eastern half of the Big Bear Lake, 100,000 scale quadrangle, California and analysis of the depths of several basins," U.S. Geol. Survey, Reston, VA, USA, Open-File Rep. 02-353, 2002. 